\title{
Index, notes et bibliographie(s). Le travail d'édition scientifique en acte
}

\section{Matthieu Béra}

\section{OpenEdition}

12 Journals

Édition électronique

URL : http://journals.openedition.org/assr/28140

DOI : 10.4000/assr.28140

ISSN : 1777-5825

Éditeur

Éditions de l'EHESS

\section{Édition imprimée}

Date de publication : 31 décembre 2016

Pagination : 153-168

ISSN : 0335-5985

\section{Référence électronique}

Matthieu Béra, «Index, notes et bibliographie(s). Le travail d'édition scientifique en acte », Archives de sciences sociales des religions [En ligne], 176 | octobre-décembre 2016, mis en ligne le 01 janvier 2019, consulté le 02 janvier 2020. URL : http://journals.openedition.org/assr/28140; DOI : 10.4000/assr. 28140 


\section{Notes critiques}





\title{
Matthieu Béra
}

\section{Index, notes et bibliographie(s). \\ Le travail d'édition scientifique en acte}

\author{
À propos de : \\ DuRKheIm Émile, Euvres. Tome I. Les Formes élémentaires de la vie \\ religieuse. Édition de Myron Achimastos, Classique Garnier, coll. \\ "Bibliothèques des sciences sociales ", 2015, 825 p. \\ ACHIMASTOS Myron, "Remarques sur l'édition critique des Formes \\ élémentaires ", Cabiers de Recherche sociologique, $\mathrm{n}^{\circ}$ 56, 2014, \\ p. 33-52.
}

Myron Achimastos, professeur associé au département de sociologie de l'Université de Crète, a travaillé huit ans sur cette édition scientifique des Formes, tout en traduisant parallèlement l'ouvrage en grec moderne (à paraître). C'est avec le soutien du co-directeur de la collection «Bibliothèques des sciences sociales » de Garnier, Philippe Steiner, qu'il propose la première édition scientifique du classique jamais conçue en France. Il s'agit d'ailleurs de la première édition scientifique d'un livre de Durkheim, qui en publia quatre de son vivant - cinq si l'on inclut sa thèse latine sur Montesquieu (1893). Alors qu'on s'apprête ici ou là ${ }^{1}$ à célébrer le centenaire de son décès, qu'on a déjà fêté ceux de ses quatre ouvrages précédents, personne n'avait proposé un tel travail éditorial en France. Il aura donc fallu attendre plus d'un siècle et un universitaire non hexagonal $^{2}$ pour que cela advienne, enfin ${ }^{3}$.

Pierre Bourdieu, qui encouragea jadis Victor Karady à rééditer certains « Textes» de Durkheim dans les années 1970, n’a-t-il pas dit que «Durkheim

1. À ce jour, un seul colloque est annoncé pour juin 2017 par le Centre Durkheim à Bordeaux.

2. M. Achimastos a publié un article dans L'Année sociologique en 2012 dans le numéro centenaire coordonné par Massimo Borlandi. Il communiqua aussi sur Strelhow au colloque de Bordeaux organisé pour le centenaire des Formes en juin 2012 et son intervention va paraitre dans M. Béra, N. Sembel (dir.) Durkheim et la religion. Les Formes élémentaires de la vie religieuse d'hier à aujourd'hui (1912-2012), Paris, Classique Garnier, automne 2016.

3. Faut-il rappeler que les biographes de Durkheim sont ou bien anglais (Steven Lukes) ou bien québécois (Marcel Fournier)? 
[était] le paria de la philosophie et de la science françaises qui l'ont traité en chien crevé » 4 ? Dans cette conférence de 1994 qu'il prononça en hommage à Mauss et qui fut éditée de manière posthume, il appelait à

une lecture qu'on accorde très souvent aux philosophes et très rarement aux sociologues [...]. Si on lisait Mauss [et Durkheim] comme on lit Wittgenstein ou Heidegger, peut-être qu'on découvrirait une profondeur chez les auteurs de sciences sociales qu'on accorde dérisoirement aux auteurs de philosophie qui ne le méritent pas toujours.

Sans adopter le ton du Bourdieu polémiste, ni tomber dans le fétichisme des textes - ce n'est certes pas le travers qu'on pourra lui imputer à lui qui s'en prenait violemment à toute forme de "textisme ${ }^{5}$ - il est temps d'encourager les lectures approfondies et rigoureuses des grands textes des "classiques des sciences sociales " ${ }^{6}$. Cette édition nous y amène, at least. C'est une étape nécessaire que l'on va donc pouvoir franchir à partir de 2016, en partant du dernier ouvrage de Durkheim ${ }^{7}$.

Bien évidemment, nous ne relirons pas ici les Formes pour en proposer un compte rendu, comme il s'en fit des dizaines depuis $1912^{8}$ - ce serait absurde. Nous renvoyons à d'autres travaux qui sont souvent devenus eux-mêmes des classiques ${ }^{9}$. Nous allons plutôt diriger notre commentaire vers le travail de l'éditeur scientifique. Cependant, pour obtenir des précisions sur sa «ligne éditoriale ", nous n'avons que deux courtes pages à nous mettre sous la dent (p. 33 sq.). C'est une déception, tant nous aurions aimé l'entendre expliciter et justifier ses choix, qu'on imagine innombrables, passionnants et complexes. Ces deux pages

4. P. Bourdieu, "Marcel Mauss, aujourd'hui ", Sociologie et sociétés, vol. XXXVI.2, 2004, p. 15. Les Textes de Durkheim ont été édités en trois volumes par V. Karady en 1975 dans la collection du "Sens commun " (dirigée par Bourdieu), après ceux de Mauss en trois volumes aussi, réunis sous le titre Euvres (1969).

5. P. Bourdieu, Science de la science et réflexivité, Paris, Raisons d'Agir, coll. "Cours et travaux ", 2001.

6. Sur ces questions de rapport à la philosophie, voir G. Paoletti, Durkheim et la philosophie. Représentation, réalité et lien social, Paris, Classiques Garnier, coll. «Bibliothèques des sciences sociales ", 2012 ; J.-L. Fabiani, La République des philosophes, Paris, Minuit, coll. «Sens commun ", 1988.

7. Il semble que Garnier s'engage dans l'édition scientifique des quatre ouvrages de Durkheim en intitulant ce volume Euvres. Tome I. La suite viendra, et sans doute assez vite, avant la parution des Euvres complètes annoncées chez Droz depuis des années et dirigées par M. Borlandi, professeur de sociologie à la retraite de l'Université de Turin. Il y aura concurrence entre ces éditions critiques. C’est un moindre mal, après cent ans de néant.

8. Pour une liste et une description des comptes rendus, nous renvoyons au numéro des Archives de Sciences Sociales des Religions ( ${ }^{\circ} 159$, juillet-septembre 2012) sur la réception des Formes coordonné par P. Lassave et à l'article de S. Baciocchi, « Document. Les recensions des Formes du vivant de leur auteur (1912-1917) ", p. 17-27.

9. Cf. le numéro de L’Année sociologique 2012, coordonné par M. Borlandi ; Archives de sciences sociales des religions, note précédente; Cahiers de recherche sociologique, $\mathrm{n}^{\circ} 56$, printemps 2014; M. Béra et N. Sembel (dir.), op. cit., qui rassemble les contributions de M. Achimastos, J.-C. Marcel, R. Benthien, C.-H. Cuin, F. Lebaron, D. Lapeyronnie, M. Fournier, N. Sembel, W. Watts Miller, F. Rosa, P. Dirkx, F. Pizarro et moi-même. 
sèches ne nous apprennent pas grand-chose ${ }^{10}$, hormis le fait qu'Achimastos explique qu'il est parti de l'édition de 1912 au lieu de la seconde (1925), posthume, qui était truffée de fautes de frappe et de sens. Elle servit pourtant de base à toutes les "rééditions " ${ }^{11}$ françaises (1937, 1960, 1985, 1991 et 2007), ainsi qu'à toutes les traductions, chacune ayant ajouté son lot de nouvelles erreurs aux précédentes ${ }^{12}$. Pour en apprendre davantage, nous nous sommes reportés à l'article qu'Achimastos avait bien voulu rédiger à notre invitation en 2014 et qui a paru depuis dans les Cahiers de recherche sociologique. Nous y trouverons une vingtaine de pages dans lesquelles il prend le temps d'expliciter certaines options éditoriales essentielles.

Dernière remarque avant de rentrer dans le vif : si nous proposons une longue " note ", c'est qu'il nous a semblé qu'il fallait absolument consacrer à cette édition davantage qu'une recension - ou pire, une mention -, tant l'événement risquait de passer inaperçu, ce qui semble déjà un peu le cas, malheureusement ${ }^{13}$. Nous allons prendre le temps de nous intéresser tout spécialement aux références de Durkheim, dans la mesure où le travail de l'éditeur scientifique s'est focalisé sur cet aspect, que ce soit au niveau des notes, des citations, de la bibliographie et des index des noms et des notions. C'est à ce "paratexte» (Gérard Genette) que nous attacherons de l'importance dans ce commentaire, puisque c'est lui qui porte la marque du travail scientifique de l' «éditeur».

\section{Le travail éditorial sur la bibliographie}

La bibliographie de Durkheim est très abondante ${ }^{14}$ et, on s'en doute, très spécialisée. Elle est très différente de celle des ouvrages précédents, car son objet est radicalement nouveau. Il ne s'agit plus de statistiques sur le suicide (1897), d'épistémologie (1895) ou de nos sociétés « organiques » comparées aux sociétés à « solidarité mécanique » (1893), mais de religions totémiques, vestige des religions primitives ${ }^{15}$. Grâce à Achimastos - on est ici au cœur du travail d'éditeur scientifique, qui relève de la recherche bibliographique -, il est maintenant possible de réfléchir au travail bibliographique de Durkheim lui-même, à ses sources.

10. Sur 825 pages, le texte des Formes occupe $70 \%$ de la pagination. Pourquoi alors seulement deux pages sur 251, soit moins de $1 \%$ des « appendices " ?

11. Il ne faut pas confondre les "rééditions" qui sont des réimpressions auxquelles des préfaciers viennent associer leurs noms à l'ouvrage classique, avec des "éditions scientifiques " qui supposent un travail scientifique, comme leur nom l'indique.

12. La palme revient à la réédition préfacée par M. Maffesoli (éd. CNRS, 2007). Cf. le commentaire de M. Achimastos, "Remarques sur l'édition critique des Formes ", p. 33-52.

13. J'en profite pour remercier Pierre Lassave, qui a relu cette note et encouragé cette initiative.

14. Elle occupe ici une trentaine de pages (p. 705-734).

15. Le sous-titre du livre tel qu'annoncé chez Alcan en 1912 était, rappelons-le, Le système totémique en Australie (cf. "Introduction" de l'éditeur, p. 7). Le sous-titre a disparu de cette édition. On se demande pourquoi. 
Pour commencer, combien de références y trouve-t-on ? Si nos calculs sont exacts, il y aurait 305 références « directes » et 163 auteurs (on compte moins d'auteurs que de références puisque certains rassemblent sur leur nom plusieurs écrits) ${ }^{16}$. C'est à James Frazer que Durkheim a associé le plus de textes : quinze articles et ouvrages ${ }^{17}$. En outre, on découvre que $43 \%$ des références renvoient à des articles de revues. Le niveau de spécialisation est mesurable à cet indicateur. Sur les 26 périodiques recensés, 16 sont de langue anglaise.

\begin{tabular}{|c|c|c|c|c|}
\hline \multicolumn{5}{|c|}{ Tableau 1 : Périodiques référencés dans les Formes } \\
\hline Langue & Anglais & Allemand & Français & Italien \\
\hline Exemples & $\begin{array}{l}\text { - The Journal of the } \\
\text { anthropological } \\
\text { institute of Great } \\
\text { Britain and Ireland } \\
(\mathrm{n}=9) \\
\text { - The Fortnightly review } \\
(\mathrm{n}=4) \\
\text { - Folklore }(\mathrm{n}=4) \\
\text { - The American } \\
\text { Anthropologist }(\mathrm{n}=4) \\
\text { - Man }(\mathrm{n}=7)\end{array}$ & $\begin{aligned}- & \text { Globus } \\
& (\mathrm{n}=2) \\
- & \text { Anthropos } \\
& (\mathrm{n}=1)\end{aligned}$ & $\begin{array}{l}\text { - Journal } \\
\text { Asiatique } \\
\text { - Bulletin de } \\
\text { correspondance } \\
\text { bellénique }\end{array}$ & $\begin{array}{l}\text { - Rivista } \\
\text { italiana di } \\
\text { sociologia }\end{array}$ \\
\hline Nombre & 16 & 7 & 2 & 1 \\
\hline
\end{tabular}

Concernant les langues, $62 \%$ des sources sont anglaises, $25 \%$ sont françaises et $12 \%$ sont allemandes - on en trouve marginalement en latin, italien et néerlandais. Cette domination des sources anglo-américaines nous renvoie aux réponses de Durkheim à Simon Deploige quand celui-ci lui reprocha de produire une sociologie made in Germany (1907 et 1911). Durkheim lui rétorqua dans ses réponses restées célèbres qu'il ne devait quasiment rien aux Allemands ${ }^{18}$. Le quiproquo qui s'installa entre les deux auteurs venait du fait que le premier faisait mine de se reporter au Durkheim d'avant les Formes, tandis que le second songeait à ce qu'il avait pu lire et écrire sur la religion depuis 1898 , qui était devenue son objet principal. On se doute pourtant que Deploige était motivé dans ses attaques par les positions de Durkheim sur la religion. Il était assez malhonnête de sa part de ne prendre comme objet que les textes d'avant 1895, alors que son interlocuteur avait publié des éléments de sa sociologie religieuse

16. Trois occurrences pour Boas, Dorsey, W. James, Taplin; quatre pour Hill-Tout, A. Kuhn, R. Smith, Wilken ; cinq pour Hubert, Morgan; six pour N. Thomas; sept pour Howitt, Lang; huit pour R. H. Mathews ; dix pour Durkheim ; treize pour Müller ; quinze pour Frazer.

17. Pour le dire autrement : c'est aux écrits de Frazer qu'il a associé le plus souvent son propre texte.

18. Les réponses datent de 1907 et 1913. 
depuis 1898 dans L'Année sociologique ${ }^{19}$. De son côté, Durkheim cachait mal tout ce qu'il devait, de fait, à ses lectures allemandes depuis sa formation initiale jusque dans les Formes elles-mêmes : il n'est qu'à songer à l'usage intensif qu'il fait du missionnaire allemand Strelhow, l'utilisant contre Frazer ${ }^{20}$.

Pour revenir au travail de l'éditeur, on peut regretter qu'il n'existe aucun moyen de retrouver facilement une source de périodique. On ne possède pas d'index des périodiques qui renvoient, rappelons-le, à près de la moitié des références bibliographiques du livre de Durkheim. Ainsi, quand on veut chercher à quel article et auteur est associé la référence "Man, $1905 »^{21}$, il ne reste rien d'autre à faire que d'éplucher l'ensemble des références de la bibliographie! Pourquoi ne pas avoir combiné l'indexation à la bibliographie ? En effet (on y reviendra), les index renvoient aux auteurs et aux notions, pas aux sources. N'y a-t-il pas un paradoxe, ou du moins un défaut, à avoir séparé les sources bibliographiques des index ? Si les vingt-six périodiques avaient pu être adjoints à l'index des noms, par exemple, on aurait beaucoup gagné. Cela pourra-t-il être rectifié ?

\section{La bibliographie de l'éditeur}

Plus étonnante, originale et audacieuse (et donc discutable) est la bibliographie produite par Achimastos lui-même, en supplément de celle de Durkheim. Elle est loin d'être marginale: elle occupe neuf pages (p. 734-743) et ajoute 87 références et 82 auteurs. Comment l'éditeur justifie-t-il cette bibliographie " annexe » qu'il intitule "Sources et ouv[r]ages non directement référencés dans les Formes ", qui n'est donc pas celle de Durkheim, ni des Formes au sens strict?

Il n'était pas toujours suffisant de consulter les travaux auxquels Durkheim avait emprunté des arguments théoriques ou des données empiriques. Il fallait chercher, autant que faire se peut, leur source première. C’est pourquoi nous avons divisé la bibliographie en deux parties $^{22}$.

L'idée d'Achimastos fut la suivante : Durkheim utilisa souvent des références qu'il n'avait pas lues. L'éditeur se propose donc de les dévoiler pour nous, même

19. Au moment de l'attaque polémiste du professeur et prêtre (il a été ordonné en 1896) dans la revue Néo-scolastique, Durkheim venait de dispenser son troisième cours public sur la religion, à la Sorbonne (année 1906-1907), dont le résumé avait été donné dans la Revue Philosophique par l'étudiant Fontana en mai 1907. Durkheim avait déjà publié tous ses articles sur la religion dans L'Année sociologique depuis 1898.

20. Cf. la communication d'Achimastos en juin 2012 à Bordeaux et son article à paraître en 2016.

21. On sait que Durkheim a emprunté les volumes 2, 4, 5, 6, 7 pendant l'année universitaire 1908/1909 à la bibliothèque de l'ENS (cf. M. Béra, "Les emprunts parisiens de Durkheim à l'ENS et à la Sorbonne (1902-1917) ", Durkheimian Studies, 2016, à paraître). Il faut chercher longtemps pour savoir que le no 2 (1902) renvoie à un article de Powell, le no 4 (1904) à un article de Lang et le $\mathrm{n}^{\circ} 5$ (1905) à W. Thomas.

22. Cf. «Introduction" de l'éditeur, p. 33. 
si ce n'est pas toujours facile. C'est l'argument essentiel que l'on trouve dans son article de $2014^{23}$ :

Notre recherche a montré que Durkheim s'est contenté de lire la plupart des recherches ethnographiques postérieures à 1897 [i.e., à partir du lancement de l'entreprise collective bibliographique de L'Année]. Pour celles qui avaient paru avant cette date, il jugea que l'autorité de Frazer était plus que suffisante. Nous devons signaler au moins 49 références que Durkheim emprunte à Frazer sans pourtant l'avouer ${ }^{24}$.

De fait, quand on consulte les références de cette "bibliographie cachée ", on constate qu'elles sont toutes antérieures à 1897 - certaines remontent même au XVIII ${ }^{\mathrm{e}}$ siècle. Nous ne nous prononcerons pas sur la pertinence de ces références. La recherche philologique bat ici son plein et ce travail nous paraît sans limites : en référençant les sources des sources, les lectures annoncées qui ne sont pas faites, on peut aller très loin et se retrouver assez vite très seul! Cette tache érudite est impressionnante et condamnée à rester sans écho: personne n'ira l'amender ni la vérifier, en dépit des vœux pieux adressés par l'éditeur à la communauté scientifique. Nous faisons confiance au travail d'Achimastos qui a passé des années à vérifier les sources "directes " du classique et à enquêter sur ses sources «indirectes ». On attend simplement qu'il utilise lui-même ce matériau pour produire des articles qui nous apprennent quelque chose de nouveau.

\section{Le travail éditorial sur les notes}

Pas davantage que la bibliographie, les notes de bas de page de l'ouvrage de Durkheim n'ont jamais été étudiées en tant que telles, quelles que soient leurs fonctions : produire des références ou des commentaires. Le corpus est pourtant essentiel, pour qui veut se pencher sur les sources et les «postures » de l'auteur, qu'on appelle depuis Aristote en rhétorique «ethos ${ }^{25}$.

Nous passerons rapidement sur les notes du classique qui sont traitées par l'éditeur comme le texte. Cependant, à plusieurs reprises, il ne s'est pas abstenu d'intervenir à l'intérieur des notes de Durkheim, de manière discrète mais ferme : la figure des "six crochets ", notés " [...] ", lui a servi à ajouter selon une technique éditoriale classique les éléments manquants. Ainsi, les abréviations fréquentes sont rectifiées, comme suit : D[urkheim]. De plus, dans la mesure où les notes de Durkheim sont très souvent des notes de référenciation, l'éditeur est intervenu pour compléter le travail bibliographique qui était effectué de manière moins rigoureuse à cette époque. Systématiquement, l'éditeur a tenu à préciser

23. À prendre comme une sorte de making of de cette édition scientifique, on l'aura compris.

24. Cahiers de recherche sociologique, $\mathrm{n}^{\circ} 56$, p. 39.

25. Cf. M. Béra, "Présentation de soi" et rapport aux autres dans les notes de la Division du travail social de Durkheim ", in Arnould J.-C. et Poulouin C. (dir.), Notes, Rouen, Publications des Universités de Rouen et du Havre, 2008. 
entre crochets les initiales des prénoms manquants, les titres complets des ouvrages ou des revues. Quand il s'agissait de périodiques, le travail est précieux. Achimastos a dû repasser derrière chacune des références du sociologue pour inscrire ici une précision sur le numéro de la note de la page citée, là des guillemets oubliés, ou des dates de parution négligées. Ce travail de bénédictin, puisqu'il faut bien appeler les choses par leur nom, n'a pas de prix. Il est d'autant plus valeureux qu'il s'exerce quasiment de façon invisible ou silencieuse.

\section{Les notes de l'éditeur}

Il existe également des « notes de l'éditeur » entre crochets, espace traditionnellement réservé à l'expression des points de vue éditoriaux ou aux commentaires savants. Quelle est ici la nature de ces notes éditoriales ${ }^{26}$ ? Elles sont avant tout philologiques et n'offrent quasiment pas de commentaires, comme on en trouve dans certaines éditions - on pense à la Pléiade, ou à la récente traduction de Weber par Grossein ${ }^{27}$. L'éditeur y précise ce qui existe dans les éditions originales citées par Durkheim. Au besoin, il corrige, en nous donnant l'extrait vérifié. Parfois, ces notes apportent de courtes précisions biographiques - cela reste exceptionnel $^{28}$. Il est rarissime qu'Achimastos s'autorise à commenter - et critiquer - Durkheim dans ses choix, ses coupures, ses sélections - dans la mesure où «citer, c'est couper " ${ }^{29}$. Une exception : il écrit que «D. ne tient pas compte du fait que Tylor soutient son point de vue en renvoyant à Hume et Comte » (p. 119). Il n'en dit pas davantage ici. Il faut se reporter à son article de 2014 (p. 44) pour avoir des hypothèses sur ce silence : "Non seulement l'économie des Formes lui impose d'éviter une discussion théorique sur les thèses exactes de Hume et Comte, mais plus encore il voulait déprécier l'analyse de Tylor en lui reprochant un certain simplisme. »

On voit bien, par ce petit exemple, que le commentaire éditorial renvoie à des hypothèses plus ou moins démontrables. Le travail philologique se déplace alors d'une pratique objective - tel extrait renvoie à telle édition et à tel passage -

26. Elles figurent à la fin de chaque chapitre des Formes et sont numérotées par ordre alphabétique, ce qui les distingue de celles de Durkheim qui sont numérotées en chiffres et placées en bas de page.

27. M. Weber, Concepts fondamentaux de sociologie, textes choisis, traduits de l'allemand et introduits par J.-P. Grossein, Paris, Gallimard, coll. "Tel », 2016. Pendant près de cent pages, le traducteur présente le travail de Weber et se pose comme auteur : "Leçon de méthode wébérienne" (p. 9-90 + notes p. 348-379). Il adjoint à sa traduction un appareil de notes personnelles, qu'il distingue de celles de Weber par la mention N.d.T. (p. 381-396). On connaît par ailleurs Grossein pour ses prises de position virulentes contre les " mauvais » traducteurs de Weber.

28. Par exemple, note «c », p. 59 sur Wundt et le voyage de Durkheim en Allemagne en 1886 .

29. Cf. A. Compagnon, La Seconde main ou le travail de la citation, Paris, Éditions du Seuil, coll. « Points. Essais », 516 p., 2016 [1979]. 
à une pratique interprétative : "il se pourrait que Durkheim ait choisi cela, parce que... ». Cette seconde posture gêne visiblement Achimastos qui s'y aventure très rarement - on peut le comprendre. Dans le genre, on trouvera un commentaire à portée générale qui surprendra le lecteur attentif : "Comme il apparaîtra par la suite, il est très rare que D. mentionne la source réelle des informations qu'il utilise $^{30}$. " Confrontés à ce genre de commentaire, rendu possible par une proximité inégalée avec Durkheim et sa bibliographie, on aimerait évidemment en savoir plus. On aimerait qu'Achimatos généralise sur la manière de lire de Durkheim ${ }^{31}$. S'il s'avérait que Durkheim cite en déformant, emprunte en tronquant ${ }^{32}$, on aurait une vision nette de sa manière de travailler et de lire les autres. Ce "bricolage " intime, ce jeu qui s'opère en secret sur la pensée des autres ${ }^{33}$, pourrait déboucher sur des études nouvelles et passionnantes ${ }^{34}$. On en revient toujours à ce que Michel de Certeau esquissa dans ses écrits sur «l'activité lisante » quand il parlait d'un «bricolage » - il empruntait explicitement l'image à Lévi-Strauss - ou mieux, d'un «braconnage ", terme qui donne le titre à son article. L'idée est radicale ; elle a le mérite de décrire ce que "lire» veut parfois dire ${ }^{35}$.

Cependant, la plupart des «notes-à-commentaires » de l'éditeur a été donné. L'ensemble est le plus souvent ficelé par la formule réécrite cent fois : "L'original cite cette référence comme suit : [...] ». Achimastos se cantonne presque toujours au cœur du métier philologique, dans sa version objective.

\section{Le travail éditorial sur les index}

Il existe enfin un troisième lieu d'intervention éditoriale : les index. Ceuxci ont la particularité d'être totalement nouveaux, puisqu'aucun des ouvrages antécédents - qu'il s'agisse de l'édition de 1912 ou de toutes les rééditions successives $^{36}$ - n'en comporte. Seules quelques éditions traduites en ont. Il fallait par exemple se référer à l'édition italienne. Les éditeurs estimaient peut-être jusqu'à présent que les tables des "matières" détaillées et rédigées de l'époque suffisaient ${ }^{37}$. C'était confondre les « matières » avec les « notions » et les « auteurs ".

30. Note «f ", p. 120.

31. Cf. l'article de Borlandi sur le Suicide (2000, Presses universitaires de France) et du même celui sur le rapport de Durkheim à Spencer dans le collectif sur la Division du travail (Presses universitaires de France, 1993). Cf. aussi ce que dit D. Merllié sur les lectures tendancieuses de Durkheim des textes de Lévy-Bruhl.

32. Note «a ", p. 192 : "Notons que cette citation tronquée ne reflète pas exactement la pensée de Cicéron ».

33. Cf. M. Picard, La lecture comme jeu, Paris, Minuit, 1986.

34. Cf. A. Compagnon, op. cit.

35. Cf. notre article à paraître dans L’Année sociologique, 2017, « La représentation disciplinaire du social dans les références et les lectures du jeune Durkheim (1879-1894) ».

36. On voit à quel niveau d'indigence éditoriale on en est réduit depuis plus de cent ans : ni index des noms d'auteurs, ni index des matières dans aucun des ouvrages de Durkheim.

37. Voir mon article "La table des Formes ", à paraître in Mathieu G. et Arnould J.-C. (dir.), Les Tables, Paris, Classique Garnier. J’y démontre qu'on y repère des traces du « style » 


\section{L'index nominum}

Nous passerons assez vite sur l'index des auteurs, intitulé «Index des noms. Notices biographiques » (p. 745-768), qui a dû coûter de longues heures de labeur à notre éditeur, qui est parvenu à identifier tous les auteurs cités par Durkheim, directement ou non (cf. supra les bibliographies). Au total, on découvre 332 noms et autant de courtes notices biographiques. Cet "index à notices » est intéressant pour les homonymes (il existe par exemple quatre Smith, p. $763^{38}$ ). Il reste une énigme : 332 notices, c'est davantage que ce à quoi aboutissent les deux bibliographies réunies $(163+82=245)$, alors que tous les noms de l'index renvoient bien à des pages du livre. La seule explication à cette "surabondance » provient peut-être du fait que Durkheim mentionnait des noms sans les rattacher à une publication. Ou encore, autre hypothèse, l'index renvoie à des auteurs (exemple : Spinoza) qui sont cités par l'éditeur dans ses propres notes. Il ne s'agit donc pas, si l'on veut être puriste ${ }^{39}$, de l'index du texte de Durkheim, mais de l'index de cette édition conçu comme un tout, " annexes " incluses (c'est-à-dire " bibliographies + notes de l'éditeur + comptes rendus de L'Année »). Le choix nous paraît regrettable, voici pourquoi : si l'index avait été dévidé des adjonctions de son éditeur scientifique et de tout son travail philologique, il aurait permis de travailler sur les auteurs de Durkheim. Est-il possible de le faire dès lors qu'il renvoie à d'autres auteurs ? Il est évidemment envisageable d'effectuer certaines opérations pour retrouver les auteurs de Durkheim, en excluant les renvois aux pages qui viennent après les « annexes " de l'éditeur. Mais cela ne suffira pas : les notes éditoriales courent sur l'ensemble de l'ouvrage, à la fin de chaque chapitre et en notes et "six crochets ». Au final, on ne peut donc pas connaitre avec exactitude quels sont les auteurs les plus référencés ou cités ${ }^{40}$ par Durkheim ${ }^{41}$.

personnel de Durkheim, ce qui interdit de penser qu'il s'agit d'une simple table automatique et impersonnelle. La table des matières n'est pas un résumé (car elle annonce plutôt qu'elle ne délivre des informations), ni un plan détaillé, ni assurément un substitut d'index (on serait bien en peine d'y retrouver les auteurs et notions). C'est un espace auctorial, en partie destiné à la vente, chargé de donner envie à celui qui le feuillette. Smith.

38. Ce qui n'est pas inutile dans la mesure où l'on connait l'importance de Robertson

39. Cette édition y incite.

40. Les plus " indexés » sont Boas, Codrington, Curr, Dawson, Dorsey, Eylmann, Fison, Flechter, Gason, Hill-Tout, Hubert, Lang, Mathews, Mauss, Morgan, Parker, Roth, Smith, Smyth, Swanton, Thomas, Tylor, Woods. Les palmes revenant à Durkheim, Frazer, Howitt, Stelhow, Spencer et Gillen.

41. Ajoutons que l'éditeur n'a pas précisé le mode de calcul des occurrences: il s'est contenté de préciser les pages où ils sont nommés. Il s'agit là de détails, certes, mais ils dissuadent celui qui cherche à connaître le nombre exact d'auteurs " cités » ou " référencés » par Durkheim. 


\section{L'index rerum}

L'une des valeurs ajoutées principales de cette édition réside selon nous dans son index des notions, intitulé "Index des concepts» (p. 769-821). Ces cinquante pages précieuses, invisibles pour les débutants et essentielles pour les professionnels, amènent à poser sur les Formes un regard nouveau, sans même avoir à repasser par le texte ${ }^{42}$. Grâce à cet index rerum, on peut se diriger vers une analyse notionnelle des Formes. Nous nous sommes essayés à regrouper ces 194 notions, rangées comme il se doit par ordre alphabétique - sans ordre intellectuel, donc - dans le but de "synthétiser » la nomenclature, comme on agrège les données brutes pour aboutir à des « méta catégories " ${ }^{43}$. Au terme de ce petit exercice, il apparaît que l'index peut être ramené à une dizaine de " noyaux notionnels». Voici le résultat de cette opération présenté sous forme de tableau ( $c f$. ci-contre).

Ces regroupements sont indicatifs et issus d'un premier tri. Quelques termes ressortent à plusieurs catégories, par exemple celui d'" effervescence " qui renvoie à la fois à un "phénomène " et à un "groupement". Quoi qu'il en soit, cet index des notions constitue un outil de travail indispensable pour penser autrement le texte classique de Durkheim. Il ouvre des horizons sur le point de vue de son auteur.

\section{Les « annexes " éditoriales: quinze comptes rendus de Durkheim à l'année}

Il existe encore une dernière rubrique qui porte la marque du travail éditorial. Dans les «Annexes » de cette édition, bizarrement insérées entre le texte et la bibliographie de Durkheim ${ }^{44}$, Achimastos a choisi de rééditer quinze comptes rendus parus dans L'Année sociologique. Loin d'être marginale en volume, cette partie concerne tout de même une centaine de pages (p. 607-703), soit $12 \%$ de l'ensemble. On pourra discuter de la pertinence de cet " appendice ", aujourd'hui que les volumes de L'Année sont disponibles en ligne sur Gallica en quelques clics. Cependant, l'éditeur s'en justifie (p. 30-33) en expliquant que «certains comptes rendus ont joué un rôle particulier dans l'élaboration des Formes ». On veut bien le croire puisque nous avions nous-mêmes travaillé sur ce sujet ${ }^{45}$.

42. On ne sait pas comment Achimastos a choisi de faire figurer telle ou telle notion dans l'index ; on n'ira pas le taquiner sur les manques, il en existe forcément ( corps ", " art "), ni sur ce qui constitue à notre sens de fausses "notions ", parce que trop générales pour être utilisables (" méthode ", "social ", " religieux »).

43. Cf. la nomenclature des PCS de l'INSEE, qui nous font passer de 455 professions à 32 catégories et 6 groupes.

44. La bibliographie de Durkheim se retrouve donc transformée en " annexe »!

45. Cf. notre intervention au colloque de Bordeaux de juin 2012 : nous avions essayé de repérer les comptes rendus qui avaient été réutilisés dans les Formes. Cf. "Traces de L'Année dans les Formes ", in Béra M., Sembel N. (dir.), op. cit. Article pour lequel j'avais sollicité la relecture d'Achimastos, que je remercie, bien évidemment. 


\begin{tabular}{|c|c|c|}
\hline \multicolumn{3}{|c|}{ Tableau 2 : Noyaux notionnels (d'après l'index des Formes) } \\
\hline & « Noyaux notionnels» agrégés & Notions analytiques de l'index rerum \\
\hline 1 & $\begin{array}{l}\text { Croyances } \\
\text { et représentations }\end{array}$ & $\begin{array}{l}\text { Âme, cosmologie, croyances, culte, esprit, } \\
\text { divinité, emblème, idéal, imagination, } \\
\text { mythe, prohibition, profane/sacré, symbole, } \\
\text { spirituel, totem }\end{array}$ \\
\hline 2 & Pratiques et rites & $\begin{array}{l}\text { Acte magique, cérémonie, droit, empirique } \\
\text { expérience, interdiction, interdit, pratique, } \\
\text { rite, sacrifice, prohibition, sensation }\end{array}$ \\
\hline 3 & $\begin{array}{l}\text { Organisation, groupes, } \\
\text { institutions } \\
\text { (= morphologie sociale) }\end{array}$ & $\begin{array}{l}\text { Assemblée, association, autorité, civilisation } \\
\text { clan, classe collectif, collectivité, } \\
\text { communauté, droit, Église, groupe, } \\
\text { groupement, homme individuel langage } \\
\text { langue mâles organisation pouvoir, social, } \\
\text { société, solidaire }\end{array}$ \\
\hline 4 & $\begin{array}{l}\text { Logique } \\
\text { (opérations mentales) }\end{array}$ & $\begin{array}{l}\text { Associations d'idées, axiome, catégories, } \\
\text { apriorisme, causalité, notion, classe, cause, } \\
\text { concept, classification, classer, pensée, } \\
\text { concept, connaissance, conscience, } \\
\text { empirisme, espace, explication, genre, } \\
\text { idéalisation, raison, rationalité, inter- } \\
\text { prétation, pensée méthodique, idée logique }\end{array}$ \\
\hline 5 & Psychologie & $\begin{array}{l}\text { Association d'idée, conscience, délire, } \\
\text { entendement, esprit, idéalisation, } \\
\text { imagination, mental, inconscient, sensation, } \\
\text { sensibilité morale }\end{array}$ \\
\hline 6 & Morale & Règle, droit, moral, idéal \\
\hline 7 & Disciplines & $\begin{array}{l}\text { Science économique, droit, esthétique, } \\
\text { géographie, histoire, philologie, philosophie, } \\
\text { psychologie, logique, ethnographie }\end{array}$ \\
\hline 8 & $\begin{array}{l}\text { Phénomènes, ou des processus } \\
\text { dynamiques }\end{array}$ & $\begin{array}{l}\text { Association, contagiosité effervescence } \\
\text { force, individuation, magique }\end{array}$ \\
\hline 9 & Religions & $\begin{array}{l}\text { Bouddhisme, christianisme, jainisme, Dieu, } \\
\text { divinité }\end{array}$ \\
\hline
\end{tabular}

C'est une chose de rappeler l'importance de l'activité de reviewer de Durkheim (et de Mauss et Hubert), qui l'occupa pendant des années à plein temps, puisqu'il fit plus de 500 recensions ${ }^{46}$. Cependant, à ce compte, on n'en finirait pas d'inclure les articles écrits par Durkheim qui lui ont "servi " à composer le texte final : pourquoi alors ne pas avoir réédité les deux articles ${ }^{47}$ si importants

46. M. Béra, «Les comptes rendus de Durkheim à L’Année sociologique. Entre certitudes et incertitudes, polémiques et controverses ", Contextes, 10, 2012.

47. Revue de métaphysique et de morale (1909) et Revne philosophique. 
mobilisés par l'éditeur en introduction, qu'il exhume et compare en détail avec le texte final des Formes ? Ceux-là n'ont jamais été réédités et sont difficilement accessibles sur internet. Pourquoi ne pas avoir renvoyé aussi aux comptes rendus que Mauss fit "pour» Durkheim ? Son travail de recension, aussi déterminant que son travail théorique, aurait aussi pu être réédité. Honnêtement, on l'aura compris, cette annexe nous paraît discutable, à cette place et au-delà : elle mériterait autre chose. On pense à un travail d'édition spécifique. Une fois de plus, on se retrouve dans cette situation : les recensions de Durkheim sont rééditées par morceaux, découpées, morcelées, comme dans les Textes de Karady en 1975 chez Minuit, dans l'anthologie supervisée par Jean Duvignaud en 1969 dans Journal sociologique (Presses universitaires de France) ou dans les rééditions de Nandan (1977). Ce n'est certainement pas le meilleur service à leur rendre. Leur mention et analyse en introduction aurait suffi.

\section{Remarques finales}

Au-delà de notre enthousiasme bien compris pour cet événement éditorial, quelles remarques pouvons-nous adresser que nous n'aurions pas encore faites ? La première est triviale. Elle exprime un regret, auquel s'adjoint une crainte. Regret que le prix de l'ouvrage soit si élevé, crainte qu'il reste hors de portée des lecteurs - y compris et surtout les étudiants qui ne dépensent presque plus rien pour les livres. Les lecteurs des Formes risquent de continuer à se diriger vers les éditions antécédentes, moins onéreuses, indigentes et lacunaires. Une collection de poche ne peut-elle être rapidement envisagée par Garnier ? La seconde remarque prolonge la précédente par un petit découragement : pourquoi avoir renoncé à recenser les fautes et monstruosités qui sont diffusées dans les éditions circulantes, alors que leur nombre augmente d'édition en édition - sans parler des éditions qui circulent sur le net ? On n'en connait pas l'ampleur, seul Achimastos aurait été à même de nous l'indiquer. Il n'en dit rien, par pudeur, discrétion, écœurement peut-être, on ne saurait dire ; par économie de temps certainement : quelle rentabilité aurait-il trouvé à recenser les erreurs des autres, comme il traqua des années durant celles de Durkheim ? L'inconvénient de ce silence expert est pourtant évident : en taisant le mal, on ne l'empêche pas de prospérer. Dernière remarque, qui relève d'un choix éditorial et qui nous paraît un peu maladroit: les références à la «littérature secondaire " sont presque inexistantes. Ni la longue introduction de trente pages - sur laquelle nous ne sommes pas revenus - ni l'appareil critique, à quelques rares et courtes exceptions, ne signalent quelques-uns des auteurs ayant écrit sur les Formes des choses très importantes. Quid des travaux de Steven Lukes - une seule petite note, p. 15 -, de Marcel Fournier dans les années 1990 et 2000 sur Mauss et Durkheim, des articles des Durkheimian studies lancés par Philippe Besnard en 1973 ? Quid du Durkheimian Quest (2012) de William Watts-Miller qui donne le tableau complet des plans comparés des cours de 1906 et le plan des Formes, 
des recherches indépassables de William Pickering (Durkheim on Religion, 1984) - une seule occurrence en note ? Achimastos, dans sa longue introduction sur "l'histoire et la préhistoire des Formes ", ne fait pas mieux, pourtant, que de réécrire la même chose sans signaler ces deux dernières références. Dans un autre secteur, quid des réflexions de Susan Stedman Jones sur les "catégories " (Durkheim Reconsidered, 2001) et de tous les philosophes qui débattent aujourd'hui sur le "pragmatisme» de Durkheim et son influence sur les ethnométhodologues ${ }^{48}$ ? Quid des recherches de Robert Allun Jones (The Secret of the Totem), de Frederico Rosa sur le totémisme et ses débats ? Quid des travaux de Giovanni Paoletti (Durkheim et la philosophie, 2012), des numéros spéciaux des revues de 2012 (pour le centenaire) de L’Année Sociologique, des Archives de sciences sociales des religions, des Durkheimian Studies, des Cabiers de recherche sociologique (2014), dans lesquels il a été invité lui-même à participer ? Il est pour le moins dommage que ni dans ses notes éditoriales ni dans son introduction qui retrace la préhistoire, puis l'histoire des Formes, l'éditeur qui se fait alors auteur n'ait jugé utile de se référer à quelques éléments de cette "littérature secondaire » qui porte parfois très mal son nom. L'occasion aurait été excellente de la mettre en valeur, d'en faire bénéficier les lecteurs qui ne demandent qu'à se régaler de son érudition.

\author{
Matthieu BÉRA \\ IRDAP, Université de Bordeaux \\ matthieu.bera@u-bordeaux.fr
}

48. Cf. S. Baciocchi et J.-L. Fabiani, «Durkheim's Lost Argument (1895-1955): Critical Moves on Method and Truth ", Durkheimian Studies / Études Durkheimiennes, vol. 18, 2012, p. $19-40$. 
